\title{
Exercise hemorheology: Classical data, recent findings and unresolved issues
}

\author{
Philippe Connes $^{\mathrm{a}, \mathrm{b}, *}$, Michael J. Simmonds ${ }^{\mathrm{c}}$, Jean-Frederic Brun ${ }^{\mathrm{d}}$ and Oguz K. Baskurt ${ }^{\mathrm{e}}$ \\ ${ }^{\mathrm{a}}$ UMR Inserm U665, Université des Antilles et de la Guyane, Pointe-à-Pitre, Guadeloupe \\ ${ }^{\mathrm{b}}$ Université des Antilles et de la Guyane, Laboratoire ACTES (EA 3596), Département de \\ Physiologie, Pointe-à-Pitre, Guadeloupe \\ ${ }^{\mathrm{c}}$ Faculty of Health Sciences and Medicine, Bond University, QLD, Australia \\ ${ }^{\mathrm{d}}$ Inserm U1046, Universitéde Montpellier 1, Université de Montpellier 2, Montpellier, France \\ ${ }^{\mathrm{e}}$ School of Medicine, Koc University, Istanbul, Turkey
}

\begin{abstract}
The present review focuses on the past and recent knowledge in the field of exercise hemorheology and presents some unresolved issues for opening discussion. Acute exercise is associated with a rise in hematocrit which results in an increase in blood viscosity. Whereas increased blood viscosity was previously viewed as having negative consequences for cardiovascular function and aerobic performance, recent findings suggest dynamic changes in blood viscosity might be useful for vascular function during exercise by increasing nitric oxide production. Other determinants of blood viscosity are altered by exercise (e.g., decreased red blood cell deformability, increased red blood cell aggregation and plasma viscosity) and may, independent of the associated effect on blood viscosity, directly modulate aerobic capacity. However, the data published on the effects of exercise on the hemorheology are not consistent, with some studies showing decreased, unchanged, or increased red blood cell deformability/aggregation when compared with rest. These discrepancies seem to be related to the exercise protocol investigated, the population tested or the methodogy utilized for hemorheological measurements. Finally, this review focuses on the effects of exercise training (i.e. chronic physical activity) on the hemorheological profile of healthy individuals and patients with cardiovascular and metabolic disorders.
\end{abstract}

Keywords: Exercise, training, blood viscosity, plasma viscosity, red blood cell deformability, red blood cell aggregation

\section{Introduction}

During states of increased energetic demand, a continuous rise in the rate of oxygen delivery and utilization may be observed until, at least in moderate-to-heavy intensity efforts, aerobic metabolism completely supplies the required energy cost. While highly trained individuals demonstrate faster rises in the rate of oxygen uptake, various disease states impair the capacity for performing work aerobically, when compared with untrained, healthy individuals. Consequently, the properties that influence oxygen transport and distribution (e.g., blood rheology) during exercise are important for understanding the mechanisms for reduced/improved aerobic exercise performance. Progression in hemorheological techniques improved the ability of performing blood rheological studies, and coupling hemorheological approaches with exercise physiology resulted in a renewal of interest in this scientific field. The present review will

\footnotetext{
${ }^{*}$ Corresponding author: Philippe Connes, UMR Inserm 665, Hôpital Ricou, CHU de Pointe à Pitre, 97159 Pointe à Pitre, Guadeloupe. E-mail: pconnes@yahoo.fr.
} 
address classical findings in exercise hemorheology and will present new directions. In addition, some unresolved issues will be presented for opening discussion.

\section{Acute effects of exercise on hemorheology and relationships with performance}

\subsection{Blood viscosity}

Increased blood viscosity during exercise has been well reported (see [12]) and is caused by the rise of hematocrit induced by several mechanisms including fluid shift, water loss, release of sequestered RBCs from spleen and water trapping in muscle $[2,12,22]$. It appears that moderate dehydration $(\approx 2 \%$ of weight loss) occurring during exercise has a limited impact on blood viscosity in healthy sportsmen $[1,85]$. The changes in plasma viscosity, red blood cell (RBC) deformability and aggregation during exercise may also contribute to the changes in blood viscosity. However, the hemorheological response to exercise appears to be specific to the mode and intensity of the effort, and the training status of the individual.

Cycling exercise usually increases blood viscosity by $15-20 \%$ [11-12], due to an increase in plasma viscosity $(+5-10 \%)$ and hematocrit $(+10-15 \%)$. In contrast, prolonged running exercise performed in an outdoor environment does not alter blood viscosity or hematocrit [38, 60, 84]. Senay and Pivarnik [73] proposed that blood and plasma volumes are usually well maintained during such exercise, unless athletes lose a considerable amount of water (around 4-5\% of total body weight). In addition, the footstrike hemolysis that occurs during running has been suggested to be responsible for the lack of change in the hemorheological profile observed in these studies. The most rigid RBCs could be destroyed during foostrike hemolysis, and the typical rise in hematocrit could be partially blunted [82, 84]. Further studies investigating the effects of cycling and running exercise on blood rheology in the same population (e.g., triathletes) may assist with definitively addressing this issue.

\subsection{Blood viscosity, shear rate and nitric oxide}

According to the Poiseuille law, increased blood viscosity may raise vascular resistance and increase the risks for cardiovascular complications. From an exercise physiology point of view, having high blood viscosity has been classically suspected to negatively impact on performance. Indeed, significant correlations have been reported between resting blood fluidity and indices of physical fitness such as endurance time, physical work capacity at a heart rate of $170 \mathrm{bpm}$ or maximal oxygen consumption $\left(\mathrm{VO}_{2 \max }\right)$ [12]. Brun et al. [14] proposed that decreased resting blood viscosity could improve $\mathrm{O}_{2}$ delivery to muscle during exercise in trained individuals.

During exercise, an excessive increase in blood viscosity was proposed to be potentially dangerous for the cardiovascular system because it is suspected to increase vascular resistance and increase the post-load work of the heart [28, 98]. However, blood vessels are not rigid tubes and their diameter may react and adjust in relation to the instantaneous hemorheological state. Experimental results demonstrated that increased blood viscosity may, in fact, decrease vascular resistance and increase tissue perfusion [17, 86] because the resulting increased wall shear stress may stimulate nitric oxide (NO) production by endothelial cells to create a vasodilatory compensation [69-71]. Connes et al. [27] recently studied the relationships between the changes in blood viscosity, vascular resistance, NO production and vascular hindrance in healthy sportsmen performing submaximal cycling exercise. The authors observed: 1) a 


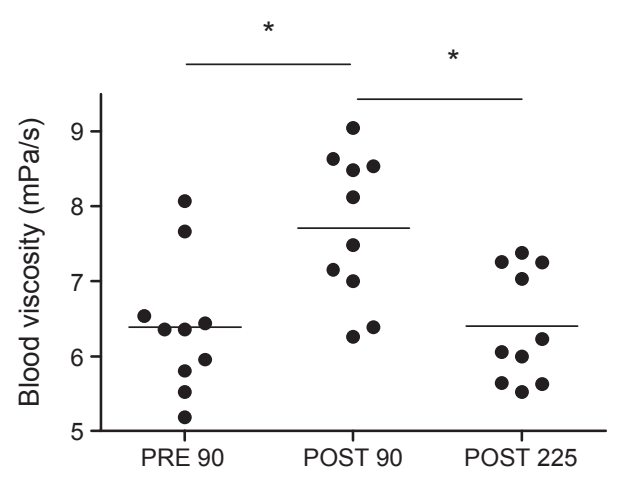

Fig. 1. Comparison between blood viscosity measured at rest at $90 \mathrm{~s}^{-1}$ (PRE 90) and blood viscosity measured at the end of a 15 min submaximal cycling exercise (105\% ventilatory threshold 1) at either $90 \mathrm{~s}^{-1}$ (POST 90) or $225 \mathrm{~s}^{-1}$ (POST 225). The data used for this figure were those from Connes et al. [27] +1 additional subject $(n=10)$. ${ }^{*}$ Statistical difference $(p<0.05)$.

positive correlation between the increase in blood viscosity and the increase in NO production during exercise; 2) a negative correlation between the increase in NO production and the decrease in vascular hindrance; 3 ) a negative correlation between the increase in blood viscosity and the decrease in systemic vascular resistance. These findings suggest that a marked rise in blood viscosity during exercise could be necessary for NO production and adequate vasodilation. We suspect that greater vasodilation could be beneficial for improving peripheral blood distribution and thus allowing for optimal aerobic performance during exercise. Having decreased resting blood viscosity could be a way to obtain the largest rise in blood viscosity in response to exercise; that could explain why decreased baseline blood viscosity is also related to improved aerobic fitness. But, of course, this compensatory response is dependant on the "health status" of the endothelium [91] and a rise in blood viscosity might be more detrimental for vascular system in the case of endothelial dysfunction [19].

When measuring the effects of exercise on blood viscosity, the results are reported and interpreted for a given shear rate. Blood is a shear-thinning fluid and its viscosity decreases when the shear rate increases. It is difficult to ascertain how blood viscosity changes measured in vitro may impact on the vascular system in vivo but the shear rate is not constant at rest and during exercise. Padilla et al. [62] reported a resting shear rate of $\approx 100 \mathrm{~s}^{-1}$ at the brachial artery level. During a semi-recumbent cycling exercise at $120 \mathrm{~W}$, the shear rate increased to $\approx 250 \mathrm{~s}^{-1}$. At the femoral artery level, Gonzales et al. [39] reported a rise from $\approx 60 \mathrm{~s}^{-1}$ at rest to $\approx 200-250 \mathrm{~s}^{-1}$ during a progressive single-leg knee extensor exercise. If we re-analyze the effects of exercise on blood viscosity taking into account the changes in shear rate occurring with exercise in the largest arteries (resting shear rate $=90 \mathrm{~s}^{-1}$; exercising shear rate $=225 \mathrm{~s}^{-1}$; Fig. 1), we observe no significant change of blood viscosity (PRE 90 vs POST 225) whereas for a given shear rate $\left(90 \mathrm{~s}^{-1}\right.$ at rest and during exercise; Fig. 1), the viscosity of blood increases (PRE 90 vs POST 90). Indeed, the effects of hematocrit increase and other RBC rheological changes on blood viscosity could be blunted in-vivo because the increase of shear rate (as a consequence of the increased blood flow) may thin blood. Nevertheless, even by taking into account the changes in shear rate occurring during exercise $\left(90 \mathrm{~s}^{-1}\right.$ to $\left.225 \mathrm{~s}^{-1}\right)$, the non-statistically significant changes in blood viscosity (Fig. 1) are still associated with the changes in NO production (Fig. 2). Both the increase in shear rate and a positive change in blood viscosity may increase shear stress to stimulate NO production. Further studies are required to study these associations in detail. In addition, it remains unknown whether the magnitudes of changes in shear rate 


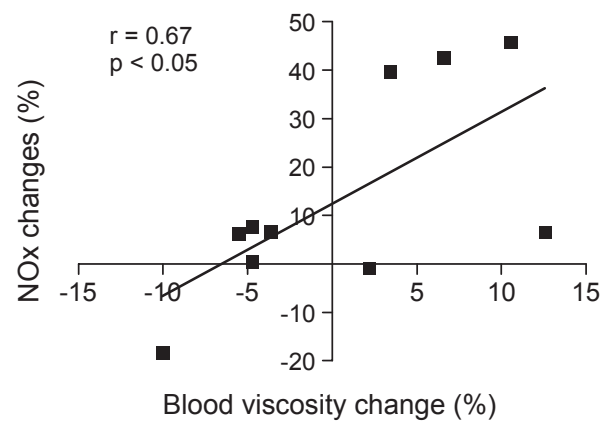

Fig. 2. Relationships between the changes in blood viscosity (taking into account the changes in shear rate occurring during exercise) and NO production (NOx) during exercise. NOx and blood viscosity were measured at rest and at the end of a $15 \mathrm{~min}$ submaximal cycling exercise (105\% VT). The changes in NOx and blood viscosity between rest and the end of exercise were calculated and plotted on this figure. To calculate the changes in blood viscosity, resting values were determined at a shear rate representing a baseline state (i.e. $90 \mathrm{~s}^{-1}$ ) and values obtained at the end of exercise were measured by using a shear rate reflecting what happens during exercise (i.e. $225 \mathrm{~s}^{-1}$ ). The data used for this figure were those from Connes et al. [27] +1 additional subject $(n=10)$.

occurring in the microcirculation during exercise are comparable with those occurring in large vessels. Further studies are needed to address this issue.

\subsection{Plasma viscosity}

When sedentary or endurance trained subjects perform short-duration maximal or submaximal exercise (mainly cycling), plasma viscosity usually increases by up to $5-10 \%$ at the end of the exercise, when compared with resting values $[13,23,34,36,81]$. This increase contributes, with the other blood rheological changes, to the increase of blood viscosity at a given shear rate during exercise. Increased plasma viscosity has been attributed to the rise in plasma protein content, such as fibrinogen, $\alpha 1$-globulins, $\alpha 2$ globulins, $\beta$-globulins and $\gamma$-globulins $[87,96]$, and probably, at least in part, to water loss. In contrast, several studies reported no change in plasma viscosity during prolonged running $[38,59,60,84]$ and the reasons are not fully understood.

\subsection{Red blood cell deformability}

The effects of acute exercise on RBC deformability have been examined in several studies, with most reporting a decrease $[13,36,38,61,67,97,98]$. This decrease may contribute to the rise in blood viscosity during exercise. Several factors might be involved in this reduction. Lactate and hydrogen ion accumulation within RBCs [53, 78] and oxidative stress [98] have been hypothesized to decrease RBC deformability during exercise. In contrast, few studies observed improved RBC deformability during acute exercise [23, 29, 43, 92]. Findings from Bor-Kucukatay et al. [9] support that NO could positively influence RBC deformability. Although very few studies investigated the effects of NO on RBC deformability during exercise, Suhr et al. [80] suggested that changes in the RBC eNO-synthase activity could positively regulate this hemorheological property. Nevertheless, findings from Bateman et al. [6] suggest that NO could decrease RBC deformability. Mesquita et al. [55] demonstrated that there 
is probably a dose-dependent effect of $\mathrm{NO}$ on $\mathrm{RBC}$ deformability with normal concentration having beneficial effect on RBC deformability whereas high concentration could be deleterious. Additional studies are clearly required to examine the relationships between RBC eNO-synthase activity or expression, and the effects of $\mathrm{NO}$ concentration on $\mathrm{RBC}$ deformability during exercise.

The mechanisms at the origin of the increase in RBC deformability during exercise are not well understood but it has been shown that a rise in lactate concentration was able to cause $\mathrm{RBC}$ rigidification in sedentary people while it resulted in RBC deformability improvement in endurance-trained athletes [24]. It seems that $\mathrm{RBC}$ from endurance sportsmen could better cope with lactate than RBC from sedentary subjects. However, a recent study in our laboratory of well-trained cyclists demonstrated that during cycling exercise that increased lactate concentration (i.e., heavy-intensity exercise), RBC deformability was decreased more than during moderate-intensity exercise that did not induce changes in lactate concentration (Simmonds et al., unpublished). Endurance training also protects RBC from oxidative damage. Yalcin et al. [97] reported that the time course of RBC deformability impairment induced by exercise differed between trained and untrained rats. The alterations in RBC deformability and RBC lipid peroxidation after $60 \mathrm{~min}$ of swimming exercise were attenuated in the trained group suggesting that physical fitness limits lipid peroxidation during exercise and the associated hemorheological alterations. Nevertheless, training and physical fitness are not sufficient to explain why RBC deformability can differentially decrease or increase with a given exercise bout, in different individuals. RBC deformability reduction has also been reported in well-trained athletes performing various physical efforts [38, 67]. Methods for RBC deformability measurements might account for some of these discrepancies with studies having used viscosimetry, filtration techniques or ektacytometry. More efforts of standardization are needed for future investigations on RBC deformability and exercise. In addition, more studies focusing on comparisons of $\mathrm{RBC}$ rheology at rest and during exercise are needed.

Impaired RBC deformability may decrease tissue perfusion and oxygenation [63]. For example, patients with sickle cell disease have very rigid RBCs which may impair blood flow into the microcirculation [3, 26]. Indeed, the surprising increase of RBC deformability found in several studies during exercise [23, $29,43,92]$ was suggested to be beneficial for tissue oxygenation and endurance performance. However, we recently reported an association between faster oxygen consumption kinetics (i.e. $\mathrm{VO}_{2}$-on kinetcis) during a rest-work transition and slightly decreased $\mathrm{RBC}$ deformability in athletes [76]. A faster $\mathrm{VO}_{2}$ on kinetics indicates a better physiological adaptation to endurance exercise [94]. We suspect that this slightly - but not pathologically - decreased RBC deformability could paradoxically improve tissue extraction of oxygen from blood due to (i). an increased transit time for RBC to pass through capillary networks [54], and; (ii). reduced distance between the RBC and vessel wall [31], which would plausibly enhance oxygen exchange between RBC and peripheral tissues. Of course, while a slight decrease in $\mathrm{RBC}$ deformability may be beneficial for enhancing oxygen extraction, it is clear that the severe RBC deformability impairment observed in several diseases such as sickle cell disease [83] or diabetes [72] is deleterious.

\subsection{Red blood cell aggregation}

A growing interest has been recently devoted to the study of RBC aggregation and its impact on hemodynamics [5]. In addition, several studies reported association between RBC aggregation abnormalities and diseases, probably as a consequence of inflammation and/or metabolic disturbances, such as in primary open-angle glaucoma [56], type 2 diabetes [75], insulin resistance [15, 16], sickle cell disease $[49,83,93]$ and others $[32,48,58,79,95]$. Few studies have investigated RBC aggregation 
in response to exercise and contrasting results have been reported: some studies described no change $[25,84,89]$, an increase, in association or not with a rise in plasma fibrinogen level $[18,36,84]$, or a delayed decrease [98] with exercise. Such findings should be considered in the context that in vitro assessment of RBC aggregation is typically performed on blood samples adjusted to standardised hematocrit (usually 40\%); increased hematocrit observed during exercise likely increases RBC aggregation in vivo due to an increased cell-cell contact. Nevertheless, the reasons for incongruent reports are unclear, but may be related to the differences in: 1) the population tested (athletes or sedentary subjects, population with disease); 2) the exercise performed (cycling, running, resistance exercise); 3) the technique used to measure aggregation (backscattered technique versus light transmission technique).

\section{Training effects on hemorheology}

\subsection{Healthy population}

Whereas acute exercise may increase blood viscosity at a given shear rate, chronic exercise (endurance or resistance exercise) usually results in a decrease [12, 46, 68]. Following a cessation of exercise (few hours or few days later), there is an increase in plasma volume [12] which represents a reversal of the acute hyperviscosity observed during acute exercise, resulting in an "autohemodilution" $[35,36]$. The repetition of exercise during consecutive days leads to a chronic "autohemodilution" resulting in a low baseline hematocrit - low baseline viscosity patterns $[12,35,46]$. Plasma volume expansion is also responsible for the decrease of the plasma viscosity contributing to the decrease of blood viscosity [35]. In contrast, overtraining syndrome is associated with a reversal of the "autohemodilution" phenomenon with hematocrit, plasma and blood viscosities increasing above levels observed in the trained state $[88,90]$. The mild chronic inflammation associated with overtraining could be responsible for these hemorheological changes [90].

Exercise training also induces adaptations of RBC aggregation [46] and RBC deformability [14]. Ernst et al. [35] reported increased RBC deformability in athletes compared to sedentary subjects, a finding later confirmed by Smith et al. [77]. A 3-month longitudinal study of initially untrained healthy volunteers performing regular training also revealed a fall of blood viscosity and an improvement of RBC deformability [35]. The reasons of the RBC deformability improvement in healthy population under training have been rarely addressed but Smith et al. [77] found higher proportion of young deformable RBCs in athletes than in untrained subjects. The higher turnover of RBCs and greater elimination of old fragile RBCs in physically active individuals might account for this finding. The production of RBCs is under the control of erythropoietin (EPO), which is up-regulated with regular exercise training [44]. It is not surprising, therefore, that Zhao et al. [99] demonstrated that increased RBC deformability in endurance-trained rats involved erythropoiesis. Specifically, exercise training caused a rise of circulating EPO which was associated with increased RBC deformability. The increased RBC deformability observed in athletes has been hypothesized to contribute, in part, to improved endurance sport performance $[22,77]$.

\subsection{Cardiovascular diseases}

Diseases related to the macrovasculature and microvasculature represent the principal causes of death and disability in many countries. Accumulating evidence suggests that hemorheological disturbances 
(e.g., elevated plasma and whole blood viscosity, RBC aggregation, plasma fibrinogen concentration) are integral in the development of cardiovascular disorders [47]. Kesmarky et al. [45] reported that ischemic heart disease patients presented with increased hematocrit, plasma and whole blood viscosity, and plasma fibrinogen concentration, compared with healthy controls. Moreover, the degree of hemorheological impairment was related to the number of coronary vessels with angiographic evidence of significant lesions. While the efficacy of exercise therapy in cardiac rehabilitation is clear, with significant improvements in body composition, aerobic capacity, blood chemistries and reduced health expenditure, it is less clear of the effects of exercise training on "hemorheological health" in patients with cardiovascular diseases.

Acute exercise in patients with coronary artery disease invokes what could be considered negative consequences: increased plasma viscosity, von Willebrand factor and plasma fibrinogen concentration [51]. The effect of chronic exercise in patients with cardiovascular diseases is unclear, due to conflicting reports. Ernst and Matrai [37] reported that treadmill walking $5 \mathrm{~d} / \mathrm{wk}$ in claudication patients decreased blood and plasma viscosity, decreased RBC aggregation, and increased RBC filterability within $4 \mathrm{wk}$. Daily walking and regular cycling ergometry were ineffective, however, for improving whole blood or plasma viscosity in stable post myocardial infarct patients [66]. Levine et al. [52] investigated the effect of 10-wk cardiac rehabilitation that included 30-40 min of moderate-intensity exercise on $3 \mathrm{~d} / \mathrm{wk}$. This training program failed to improve hemorheological indices when compared with baseline values. In contrast, Church et al. [21] reported that a 12-wk cardiac rehabilitation program incorporating moderateintensity exercise and dietary education, significantly decreased plasma and blood viscosity. Lack of consistent findings are likely due to differences in hemorheological techniques (e.g., tube versus rotational viscometry) and study design; for instance, the studies reporting positive effects of exercise training employed high-intensity exercise efforts [37], or provided dietary intervention in addition to exercise therapy [21].

The lack of well-designed studies investigating the effect of regular exercise on blood rheology in cardiovascular diseases limits the capacity to make definite comment regarding its efficacy. Nevertheless, the widely reported increased peak aerobic capacity following exercise training, in patients with cardiovascular diseases, coincides with increased maximal cardiac output yet unaltered mean aortic pressures: this indicates that peripheral resistance must also decrease. Hambrecht et al. [42] reported that 6-months of regular cycle ergometry reduced total peripheral resistance by $\sim 20 \%$ in patients with chronic heart failure. Increased conductance of the vasculature is often reported following exercise training, but is likely to be endothelial-dependent and highly localized within the trained limb [40]. Moreover, recent evidence suggests that while regular exercise improved vascular function in healthy subjects, the resting diameter of vessels remained unchanged and only vessels that experienced high shears during exercise training demonstrated improvements in function [8]. Previous findings corroborate that exercise training in coronary artery bypass candidates significantly increased endothelium-dependent vasodilatory capacity and average peak blood-flow velocity of left internal mammary artery in vivo, when compared with sedentary, matched candidates [41]. Specimens of left internal mammary artery obtained during subsequent bypass surgery indicated that exercise training increased mRNA and protein expression of eNOS, and increased the ratio of phosphorylated versus unphosphorylated eNOS; importantly, shear-stress dependent mechanisms (i.e., Akt-dependent and Ser ${ }^{177}$ ) explained the increased phosphorylation state of eNOS [41], highlighting the link between blood rheological properties (which directly influence shear stress), exercise, and vascular function. Thus, while exercise training studies utilizing hemorheology techniques are limited in cardiovascular disease patients, careful consideration of the literature implicates rheological properties of blood to be complicit with the often cited benefits following exercise training. 


\subsection{Metabolic disease}

Metabolic diseases include a wide-spectrum of disorders stemming from autoimmune, lifestyle and genetic sources. While diverse in etiology for onset and progression, remarkable cross-over may be observed in the manifestations of the co-morbidities and complications of metabolic disorders. For instance, while type 1 and type 2 diabetes mellitus result from vastly different etiologies (autoimmune and environmental, respectively), the resultant complications are closely related (e.g., retinopathies; neuropathies; nephropathy; cardiovascular diseases). Perhaps not coincidently, localized hypoxia has been implicated in the progression of many complications associated with metabolic disorders. Given microcirculatory blood flow is balanced by vascular tone and the rheological properties of blood, the importance of hemorheological health is obvious in the development and progression of hypoxia-related complications.

Cross-sectional studies in high-risk populations for metabolic disorders (e.g., obesity) provide evidence that hemorheological disturbances are associated with progression towards overt disease $[10,57,65]$. Whether exercise training may reverse or diminish the effects of impaired hemorheology in high-risk individuals seems to depend upon study design. While $10 \mathrm{~d}$ of aerobic exercise had no effect on arterial function of obese subjects with metabolic syndrome [7], $8 \mathrm{wk}$ of moderate-intensity exercise by obese and insulin resistant individuals decreased plasma viscosity; however, blood viscosity, plasma fibrinogen, RBC aggregation and RBC deformability remained unchanged [33]. Coppola et al. [30] reported that twelve wk of moderate-intensity treadmill exercise reduced blood viscosity at high $\left(450 \mathrm{~s}^{-1}\right)$ and moderate $\left(90 \mathrm{~s}^{-1}\right)$ shear rates in older, sedentary individuals. Consequently, the limited studies investigating the effects of exercise training in individuals at risk for metabolic disorders indicates that plasma viscosity may improve within $8 \mathrm{wk}$ of aerobic exercise training, while at least 12-wk of exercise training is required to induce beneficial changes in blood viscosity. Further studies investigating the mechanisms for reduced viscosity in early-stage metabolic disorders would be useful to determine whether the typical progression toward impaired hemorheology in overt-disease states may be averted using exercise training.

Blood viscosity is increased for individuals with type 2 diabetes, when compared with healthy controls [50]. Moreover, hemorheological health is markedly worse for individuals with type 2 diabetes that present with poor glycemic control and is associated with co-morbidities [75], suggesting hyperglycemia may be involved in the processes underlying increased blood viscosity and morbidity for these patients. Chronic hyperglycaemia decreases the compliance of RBCs and reduces the repulsion induced by the negative charge of the cell surface [4,64]. Very few studies have investigated the effect of exercise training on hemorheology in type 2 diabetes. One of the few studies pertinent to type 2 diabetes demonstrated that $\sim 14$ wk of intensified glycemic management, incorporating insulin therapy, exercise training and dietary modification, reduced blood glucose concentration, blood and plasma viscosity, and RBC aggregation [20]. Given that insulin, dietary interventions and exercise training were concurrently implemented, the contribution of exercise training in reducing RBC aggregation was not discernable. Simmonds et al. [74] subsequently demonstrated that 12-wk of moderate-intensity treadmill walking for $120 \mathrm{~min} / \mathrm{wk}$ significantly decreased RBC aggregation in plasma and a plasma-free aggregating solution (dextran 70), and increased maximal elongation index (RBC deformability) in older women with type 2 diabetes. Interestingly, no changes in anthropometry (e.g., body mass index) or glycemic control (e.g., $\mathrm{HbA}_{1 \mathrm{c}}$, fasting glucose concentration) were observed during the training period, suggesting that hemorheology improved in the diabetic patients due to mechanisms independent of reduced adiposity and decreased hyperglycemia. It is likely that plasma factors may explain, in part, the reduced RBC aggregation following exercise training, as the 12-wk training in diabetic patients decreased plasma fibrinogen concentration by $-6.9 \pm 10.7 \%$ (Simmonds, unpublished finding); however, given that RBC aggregability in dextran 
70 was also decreased, it is plausible that intrinsic changes to the RBC (e.g., increased electronegative charge of RBC) also contribute to improved hemorheology following exercise training in type 2 diabetes.

\section{Conclusion}

The present review presented classic and new findings, as well as unresolved issues, in the field of exercise hemorheology. Blood rheology and vascular function interact continuously to provide adequate oxygen and nutrient delivery to tissues: they cannot be considered separately. Future studies in healthy, athletic and chronic disease populations should consider investigating blood rheology, microvascular and macrovascular function concurrently, particularly at rest, during exercise and after exercise-training programs. A full overview of cardiovascular function and hemorheological responses to exercise will assist in the optimization of disease management and athletic performance.

\section{References}

[1] S. Ahmadizad, M.S. El-Sayed and D.P. MacLaren, Effects of water intake on the responses of haemorheological variables to resistance exercise, Clin Hemorheol Microcirc 35 (2006), 317-327.

[2] S. Ahmadizad, A. Moradi, S. Nikookheslat, H. Ebrahimi, A. Rahbaran and P. Connes, Effects of age on hemorheological responses to acute endurance exercise, Clin Hemorheol Microcirc 49 (2011), 165-174.

[3] T. Alexy, S. Sangkatumvong, P. Connes, E. Pais, J. Tripette, J.C. Barthelemy, T.C. Fisher, H.J. Meiselman, M.C. Khoo and T.D. Coates, Sickle cell disease: Selected aspects of pathophysiology, autonomic nervous system function and rheological considerations in transfusion therapy, Clin Hemorheol Microcirc 44 (2010), 155-166.

[4] Y. Baba, M. Kai, S. Setoyama and S. Otsuji, The lower levels of erythrocyte surface electric charge in diabetes mellitus, Clin Chim Acta 84 (1978), 247-249.

[5] O.K. Baskurt and H.J. Meiselman, RBC aggregation: More important than RBC adhesion to endothelial cells as a determinant of in vivo blood flow in health and disease, Microcirculation 15 (2008), 585-590.

[6] R.M. Bateman, J.E. Jagger, M.D. Sharpe, M.L. Ellsworth, S. Mehta and C.G. Ellis, Erythrocyte deformability is a nitric oxide-mediated factor in decreased capillary density during sepsis, Am J Physiol Heart Circ Physiol 280 (2001), H2848-H2856.

[7] T. Baynard, R.L. Carhart Jr, R.S. Weinstock, L.L. Ploutz-Snyder and J.A. Kanaley, Short-term exercise training improves aerobic capacity with no change in arterial function in obesity, Eur J Appl Physiol 107 (2009), 299-308.

[8] G.K. Birk, E.A. Dawson, C. Atkinson, A. Haynes, N.T. Cable, D.H. Thijssen and D.J. Green, Brachial artery adaptation to lower limb exercise training: Role of shear stress, J Appl Physiol 112 (2012), 1653-1658.

[9] M. Bor-Kucukatay, R.B. Wenby, H.J. Meiselman and O.K. Baskurt, Effects of nitric oxide on red blood cell deformability, Am J Physiol Heart Circ Physiol 284 (2003), H1577-H1584.

[10] J.F. Brun, C. Dupuy-Fons, J.F. Monnier, J.P. Micallef, D. Bouix, C. Peyreigne and A. Orsetti, Blood viscosity is negatively correlated to insulin sensitivity (abstract), Biorheology 32 (1995), 387-388.

[11] J.F. Brun, C. Fons, C. Supparo, C. Mallard and A. Orsetti, Could exercise-induced increase in blood viscosity at high shear rate be entirely explained by hematocrit and plasma viscosity changes? Clin Hemorheol 13(2) (1993), 187-199.

[12] J.F. Brun, S. Khaled, E. Raynaud, D. Bouix, J.P. Micallef and A. Orsetti, The triphasic effects of exercise on blood rheology: Which relevance to physiology and pathophysiology? Clin Hemorheol Microcirc 19 (1998), 89-104.

[13] J.F. Brun, J.P. Micallef and A. Orsetti, Hemorheologic effects of light prolonged exercise, Clin Hemorheol 14 (1994), 807-818.

[14] J.F. Brun, E. Varlet-Marie, P. Connes and I. Aloulou, Hemorheological alterations related to training and overtraining, Biorheology 47 (2010), 95-115.

[15] J.F. Brun, E. Varlet-Marie and E. Raynaud de Mauverger, Relationships between insulin sensitivity measured with the oral minimal model and blood rheology, Clin Hemorheol Microcirc 51 (2012), 29-34.

[16] J.F. Brun, E. Varlet-Marie, E. Raynaud de Mauverger and J. Mercier, Minimal model-derived insulin sensitivity, insulin secretion and glucose tolerance: Relationships with blood rheology, Clin Hemorheol Microcirc 51 (2012), 21-27. 
[17] P. Cabrales, J. Martini, M. Intaglietta and A.G. Tsai, Blood viscosity maintains microvascular conditions during normovolemic anemia independent of blood oxygen-carrying capacity, Am J Physiol Heart Circ Physiol 291 (2006), H581-H590.

[18] H. Cakir-Atabek, P. Atsak, N. Gunduz and M. Bor-Kucukatay, Effects of resistance training intensity on deformability and aggregation of red blood cells, Clin Hemorheol Microcirc 41 (2009), 251-261.

[19] E.N. Chirico and V. Pialoux, Role of oxidative stress in the pathogenesis of sickle cell disease, IUBMB Life 64 (2012), $72-80$.

[20] B. Chong-Martinez, T.A. Buchanan, R.B. Wenby and H.J. Meiselman, Decreased red blood cell aggregation subsequent to improved glycaemic control in Type 2 diabetes mellitus, Diabet Med 20 (2003), 301-306.

[21] T.S. Church, C.J. Lavie, R.V. Milani and G.S. Kirby, Improvements in blood rheology after cardiac rehabilitation and exercise training in patients with coronary heart disease, Am Heart J 143 (2002), 349-355.

[22] P. Connes, Hemorheology and exercise: Effects of warm environments and potential consequences for sickle cell trait carriers, Scand J Med Sci Sports 20(Suppl 3) (2010), 48-52.

[23] P. Connes, D. Bouix, F. Durand, P. Kippelen, J. Mercier, C. Prefaut, J.F. Brun and C. Caillaud, Is hemoglobin desaturation related to blood viscosity in athletes during exercise? Int J Sports Med 25 (2004), 569-574.

[24] P. Connes, D. Bouix, G. Py, C. Prefaut, J. Mercier, J.F. Brun and C. Caillaud, Opposite effects of in vitro lactate on erythrocyte deformability in athletes and untrained subjects, Clin Hemorheol Microcirc 31 (2004), 311-318.

[25] P. Connes, C. Caillaud, G. Py, J. Mercier, O. Hue and J.F. Brun, Maximal exercise and lactate do not change red blood cell aggregation in well trained athletes, Clin Hemorheol Microcirc 36 (2007), 319-326.

[26] P. Connes, R. Machado, O. Hue and H. Reid, Exercise limitation, exercise testing and exercise recommendations in sickle cell anemia, Clin Hemorheol Microcirc 49 (2011), 151-163.

[27] P. Connes, A. Pichon, M.D. Hardy-Dessources, X. Waltz, Y. Lamarre, M.J. Simmonds and J. Tripette, Blood viscosity and hemodynamics during exercise, Clin Hemorheol Microcirc 51 (2012), 101-109.

[28] P. Connes, F. Sara, M.D. Hardy-Dessources, L. Marlin, F. Etienne, L. Larifla, C. Saint-Martin and O. Hue, Effects of short supramaximal exercise on hemorheology in sickle cell trait carriers, Eur J Appl Physiol 97 (2006), 143-150.

[29] P. Connes, J. Tripette, M. Mukisi-Mukaza, O.K. Baskurt, K. Toth, H.J. Meiselman, O. Hue and S. Antoine-Jonville, Relationships between hemodynamic, hemorheological and metabolic responses during exercise, Biorheology 46 (2009), 133-143.

[30] L. Coppola, A. Grassia, A. Coppola, G. Tondi, G. Peluso, S. Mordente and G. Gombos, Effects of a moderate-intensity aerobic program on blood viscosity, platelet aggregation and fibrinolytic balance in young and middle-aged sedentary subjects, Blood Coagul Fibrinolysis 15 (2004), 31-37.

[31] G.K. Driessen, C.W. Haest, H. Heidtmann, D. Kamp and H. Schmid-Schonbein, Effect of reduced red cell "deformability" on flow velocity in capillaries of rat mesentery, Pflugers Arch 388 (1980), 75-78.

[32] Y. Du, W. Yao, Y. Qian, M. Han, Z. Wen and L. Ma, Hemorheological changes in patients with living-donor renal transplantation, Clin Hemorheol Microcirc 47 (2011), 199-209.

[33] M. Dumortier, A. Perez-Martin, E. Pierrisnard, J. Mercier and J.F. Brun, Regular exercise $(3 \times 45 \mathrm{~min} / \mathrm{wk})$ decreases plasma viscosity in sedentary obese, insulin resistant patients parallel to an improvement in fitness and a shift in substrate oxidation balance, Clin Hemorheol Microcirc 26 (2002), 219-229.

[34] E. Ernst, Changes in blood rheology produced by exercise, J Am Med Assoc 253 (1985), 2962-2963.

[35] E. Ernst, Influence of regular physical activity on blood rheology, Eur Heart J 8(Suppl G) (1987), 59-62.

[36] E. Ernst, L. Daburger and T. Saradeth, The kinetics of blood rheology during and after prolonged standardized exercise, Clin Hemorheol 11 (1991), 429-439.

[37] E.E. Ernst and A. Matrai, Intermittent claudication, exercise, and blood rheology, Circulation 76 (1987), 1110-1114.

[38] G. Galea and R.J. Davidson, Hemorrheology of marathon running, Int J Sports Med 6 (1985), 136-138.

[39] J.U. Gonzales, B.A. Parker, S.J. Ridout, S.L. Smithmyer and D.N. Proctor, Femoral shear rate response to knee extensor exercise: An age and sex comparison, Biorheology 46 (2009), 145-154.

[40] D.J. Green, N.T. Cable, C. Fox, J.M. Rankin and R.R. Taylor, Modification of forearm resistance vessels by exercise training in young men, J Appl Physiol 77 (1994), 1829-1833.

[41] R. Hambrecht, V. Adams, S. Erbs, A. Linke, N. Krankel, Y. Shu, Y. Baither, S. Gielen, H. Thiele, J.F. Gummert, F.W. Mohr and G. Schuler, Regular physical activity improves endothelial function in patients with coronary artery disease by increasing phosphorylation of endothelial nitric oxide synthase, Circulation 107 (2003), 3152-3158.

[42] R. Hambrecht, S. Gielen, A. Linke, E. Fiehn, J. Yu, C. Walther, N. Schoene and G. Schuler, Effects of exercise training on left ventricular function and peripheral resistance in patients with chronic heart failure: A randomized trial, JAMA 283 (2000), 3095-3101. 
[43] M. Hardeman, H.P. Peters and P.T. Goedhart, Low hematocrit and plasma fibrinogen in trained athletes increase hemorheological tolerance for physical stress (Abstract), Biorheology 32 (1995), 401.

[44] M. Hu and W. Lin, Effects of exercise training on red blood cell production: Implications for anemia, Acta Haematol 127 (2012), 156-164.

[45] G. Kesmarky, K. Toth, L. Habon, G. Vajda and I. Juricskay, Hemorheological parameters in coronary artery disease, Clin Hemorheol Microcirc 18 (1998), 245-251.

[46] E. Kilic-Toprak, F. Ardic, G. Erken, F. Unver-Kocak, V. Kucukatay and M. Bor-Kucukatay, Hemorheological responses to progressive resistance exercise training in healthy young males, Med Sci Monit 18 (2012), CR351-CR360.

[47] W. Koenig and E. Ernst, The possible role of hemorheology in atherothrombogenesis, Atherosclerosis 94 (1992), 93-107.

[48] R. Kotan, N. Nemeth, F. Kiss, J. Posan, K. Miszti-Blasius, L. Toth, I. Furka, I. Miko, P. Sapy and Z. Szentkereszty, Micro-rheological changes during experimental acute pancreatitis in the rat, Clin Hemorheol Microcirc 51 (2012), 255-264. DOI: 10.3233/CH-2012-1531.

[49] Y. Lamarre, M. Romana, X. Waltz, M.L. Lalanne-Mistrih, B. Tressieres, L. Divialle-Doumdo, M.D. Hardy-Dessources, J. Vent-Schmidt, M. Petras, C. Broquere, F. Maillard, V. Tarer, M. Etienne-Julan and P. Connes, Hemorheological risk factors of acute chest syndrome and painful vaso-occlusive crisis in children with sickle cell disease, Haematologica, 2012.

[50] C. Le Devehat, M. Vimeux and T. Khodabandehlou, Blood rheology in patients with diabetes mellitus, Clin Hemorheol Microcirc 30 (2004), 297-300.

[51] K.W. Lee, A.D. Blann, J. Ingram, K. Jolly and G.Y. Lip, Incremental shuttle walking is associated with activation of haemostatic and haemorheological markers in patients with coronary artery disease: The Birmingham rehabilitation uptake maximization study (BRUM), Heart 91 (2005), 1413-1417.

[52] G.N. Levine, C. O’Malley and G.J. Balady, Exercise training and blood viscosity in patients with ischemic heart disease, Am J Cardiol 76 (1995), 80-81.

[53] V. Lipovac, M. Gavella, Z. Turck and Z. Skrabalo, Influence of lactate on the insulin action on red blood cell filterability, Clin Hemorheol 5 (1985), 421-428.

[54] H.H. Lipowsky, L.E. Cram, W. Justice and M.J. Eppihimer, Effect of erythrocyte deformability on in vivo red cell transit time and hematocrit and their correlation with in vitro filterability, Microvasc Res 46 (1993), 43-64.

[55] R. Mesquita, B. Picarra, C. Saldanha and J. Martins e Silva, Nitric oxide effects on human erythrocytes structural and functional properties-an in vitro study, Clin Hemorheol Microcirc 27 (2002), 137-147.

[56] K. Michalska-Malecka and L. Slowinska-Lozynska, Aggregation and deformability of erythrocytes in primary open-angle glaucoma (POAG); the assessment of arterial hypertension, Clin Hemorheol Microcirc 49 (2012), 1-9.

[57] A. Moan, G. Nordby, I. Os, K.I. Birkeland and S.E. Kjeldsen, Relationship between hemorrheologic factors and insulin sensitivity in healthy young men, Metabolism 43 (1994), 423-427.

[58] A.V. Muravyov, S.V. Cheporov, N.V. Kislov, S.V. Bulaeva and A.A. Maimistova, Comparative efficiency and hemorheological consequences of hemotransfusion and epoetin therapy in anemic cancer patients, Clin Hemorheol Microcirc $\mathbf{4 4}$ (2010), 115-123.

[59] D. Neuhaus, C. Behn and P. Gaehtgens, Haemorheology and exercise: Intrinsic flow properties of blood in marathon running, Int J Sports Med 13 (1992), 506-511.

[60] D. Neuhaus and P. Gaehtgens, Haemorrheology and long term exercise, Sports Med 18 (1994), 10-21.

[61] G.S. Oostenbrug, R.P. Mensink, M.R. Hardeman, T. De Vries, F. Brouns and G. Hornstra, Exercise performance, red blood cell deformability, and lipid peroxidation: Effects of fish oil and vitamin E, J Appl Physiol 83 (1997), 746-752.

[62] J. Padilla, G.H. Simmons, L.C. Vianna, M.J. Davis, M.H. Laughlin and P.J. Fadel, Brachial artery vasodilatation during prolonged lower limb exercise: Role of shear rate, Exp Physiol 96 (2011), 1019-1027.

[63] K. Parthasarathi and H.H. Lipowsky, Capillary recruitment in response to tissue hypoxia and its dependence on red blood cell deformability, Am J Physiol 277 (1999), H2145-H2157.

[64] I.K. Petropoulos, P.I. Margetis, M.H. Antonelou, J.X. Koliopoulos, S.P. Gartaganis, L.H. Margaritis and I.S. Papassideri, Structural alterations of the erythrocyte membrane proteins in diabetic retinopathy, Graefes Arch Clin Exp Ophthalmol 245 (2007), 1179-1188.

[65] E. Raynaud, J.F. Brun, A. Perez-Martin, A. Orsetti and M. Solere, Negative correlation between plasma fibrinogen and insulin sensitivity measured with the minimal model technique, Clin Hemorheol Microcirc 18 (1998), 323-330.

[66] W.H. Reinhart, G. Dziekan, U. Goebbels, J. Myers and P. Dubach, Influence of exercise training on blood viscosity in patients with coronary artery disease and impaired left ventricular function, Am Heart J 135 (1998), 379-382. 
[67] W.H. Reinhart, M. Stäubli and W. Straub, Impaired red cell filterability with elimination of old red blood cells during a 100-km race, J Appl Physiol 54 (1983), 827-830.

[68] A.J. Romain, J.F. Brun, E. Varlet-Marie and E. Raynaud de Mauverger, Effects of exercise training on blood rheology: A meta-analysis, Clin Hemorheol Microcirc 49 (2011), 199-205.

[69] B.Y. Salazar Vazquez, P. Cabrales, A.G. Tsai and M. Intaglietta, Nonlinear cardiovascular regulation consequent to changes in blood viscosity, Clin Hemorheol Microcirc 49 (2011), 29-36.

[70] B.Y. Salazar Vazquez, J. Martini, A. Chavez Negrete, A.G. Tsai, S. Forconi, P. Cabrales, P.C. Johnson and M. Intaglietta, Cardiovascular benefits in moderate increases of blood and plasma viscosity surpass those associated with lowering viscosity: Experimental and clinical evidence, Clin Hemorheol Microcirc 44 (2010), 75-85.

[71] B.Y. Salazar Vazquez, R. Wettstein, P. Cabrales, A.G. Tsai and M. Intaglietta, Microvascular experimental evidence on the relative significance of restoring oxygen carrying capacity vs. blood viscosity in shock resuscitation, Biochim Biophys Acta 1784 (2008), 1421-1427.

[72] H. Schmid-Schonbein and E. Volger, Red-cell aggregation and red-cell deformability in diabetes, Diabetes 25 (1976), 897-902.

[73] L.C. Senay Jr and J.M. Pivarnik, Fluid shifts during exercise, Exerc Sport Sci Rev 13 (1985), 335-387.

[74] M.J. Simmonds, C.L. Minahan, K.R. Serre, G.C. Gass, S.M. Marshall-Gradisnik, L.J. Haseler and S. Sabapathy, Preliminary findings in the heart rate variability and haemorheology response to varied frequency and duration of walking in women 65-74 yr with type 2 diabetes, Clin Hemorheol Microcirc 51 (2012), 87-99.

[75] M.J. Simmonds, S. Sabapathy, G.C. Gass, S.M. Marshall-Gradisnik, L.J. Haseler, R.M. Christy and C.L. Minahan, Heart rate variability is related to impaired haemorheology in older women with type 2 diabetes, Clin Hemorheol Microcirc 46 (2010), 57-68.

[76] M.J. Simmonds, J. Tripette, S. Sabapathy, S.M. Marshall-Gradisnik and P. Connes, Cardiovascular dynamics during exercise are related to blood rheology, Clin Hemorheol Microcirc 49 (2011), 231-241.

[77] J.A. Smith, D.T. Martin, R.D. Telford and S.K. Ballas, Greater erythrocyte deformability in world-class endurance athletes, Am J Physiol 276 (1999), H2188-H2193.

[78] J.A. Smith, R.D. Telford, M. Kolbuch-Braddon and M.J. Weidemann, Lactate/H+ uptake by red blood cells during exercise alters their physical properties, Eur J Appl Physiol Occup Physiol 75 (1997), 54-61.

[79] M.I. Spengler, M.J. Svetaz, M.B. Leroux, S.M. Bertoluzzo, P. Carrara, F. Van Isseldyk, D. Petrelli, F.M. Parente and P. Bosch, Erythrocyte aggregation in patients with systemic lupus erythematosus, Clin Hemorheol Microcirc 47 (2011), 279-285.

[80] F. Suhr, S. Porten, T. Hertrich, K. Brixius, A. Schmidt, P. Platen and W. Bloch, Intensive exercise induces changes of endothelial nitric oxide synthase pattern in human erythrocytes, Nitric Oxide 20 (2009), 95-103.

[81] T. Teillet, P. Pilardeau, P. Libercier, J. Vaysse and J. Hermant, Variation des protéines plasmatiques pendant un exercice de coute durée, Sci Sports 6 (1991), 173-178.

[82] R.D. Telford, G.J. Sly, A.G. Hahn, R.B. Cunningham, C. Bryant and J.A. Smith, Footstrike is the major cause of hemolysis during running, J Appl Physiol 94 (2003), 38-42.

[83] J. Tripette, T. Alexy, M.D. Hardy-Dessources, D. Mougenel, E. Beltan, T. Chalabi, R. Chout, M. Etienne-Julan, O. Hue, H.J. Meiselman and P. Connes, Red blood cell aggregation, aggregate strength and oxygen transport potential of blood are abnormal in both homozygous sickle cell anemia and sickle-hemoglobin C disease, Haematologica 94 (2009), 1060-1065.

[84] J. Tripette, M.D. Hardy-Dessources, E. Beltan, A. Sanouiller, J. Bangou, T. Chalabi, R. Chout, M. Hedreville, C. Broquere, D. Nebor, G. Dotzis, O. Hue and P. Connes, Endurance running trial in tropical environment: A blood rheological study, Clin Hemorheol Microcirc 47 (2011), 261-268.

[85] J. Tripette, G. Loko, A. Samb, B.D. Gogh, E. Sewade, D. Seck, O. Hue, M. Romana, S. Diop, M. Diaw, K. Brudey, P. Bogui, F. Cisse, M.D. Hardy-Dessources and P. Connes, Effects of hydration and dehydration on blood rheology in sickle cell trait carriers during exercise, Am J Physiol Heart Circ Physiol 299 (2010), H908-H914.

[86] A.G. Tsai, P. Cabrales and M. Intaglietta, Blood viscosity: A factor in tissue survival? Crit Care Med 33 (2005), 1662-1663.

[87] H. Vandewalle, C. Lacombe, J.C. Lelievre and C. Poirot, Blood viscosity after a 1-h submaximal exercise with and without drinking, Int J Sports Med 9 (1988), 104-107.

[88] E. Varlet-Marie, A. Gaudard, J. Mercier, F. Bressolle and J.F. Brun, Is the feeling of heavy legs in overtrained athletes related to impaired hemorheology? Clin Hemorheol Microcirc 28 (2003), 151-159.

[89] E. Varlet-Marie, A. Gaudard, J.F. Monnier, J.P. Micallef, J. Mercier, F. Bressolle and J.F. Brun, Reduction of red blood cell disaggregability during submaximal exercise: Relationship with fibrinogen levels, Clin Hemorheol Microcirc 28 (2003), $139-149$. 
[90] E. Varlet-Marie, F. Maso, G. Lac and J.F. Brun, Hemorheological disturbances in the overtraining syndrome, Clin Hemorheol Microcirc 30 (2004), 211-218.

[91] B.Y. Vazquez, M.A. Vazquez, M.G. Jaquez, A.H. Huemoeller, M. Intaglietta and P. Cabrales, Blood pressure directly correlates with blood viscosity in diabetes type 1 children but not in normals, Clin Hemorheol Microcirc 44 (2010), 55-61.

[92] P. Wahl, W. Bloch, J. Mester, D.P. Born and B. Sperlich, Effects of different levels of compression during sub-maximal and high-intensity exercise on erythrocyte deformability, Eur J Appl Physiol 112 (2012), 2163-2169.

[93] X. Waltz, M. Hedreville, S. Sinnapah, Y. Lamarre, V. Soter, N. Lemonne, M. Etienne-Julan, E. Beltan, T. Chalabi, R. Chout, O. Hue, D. Mougenel, M.D. Hardy-Dessources and P. Connes, Delayed beneficial effect of acute exercise on red blood cell aggregate strength in patients with sickle cell anemia, Clin Hemorheol Microcirc 52 (2012), 15-26.

[94] B.J. Whipp, The slow component of $\mathrm{O}_{2}$ uptake kinetics during heavy exercise, Med Sci Sports Exerc 26 (1994), 1319-1326.

[95] M. Wiewiora, K. Sosada, L. Slowinska, J. Piecuch, M. Gluck, W. Zurawinski and B. Turczynski, Sex-dependent differences in rheological properties and the relation of blood viscosity to erythrocyte aggregation indices among morbidly obese patients, Clin Hemorheol Microcirc 44 (2010), 259-267.

[96] S.C. Wood, M.P. Doyle and O. Appenzeller, Effects of endurance training and long distance running on blood viscosity, Med Sci Sports Exerc 3 (1991), 1265-1269.

[97] O. Yalcin, M. Bor-Kucukatay, U.K. Senturk and O.K. Baskurt, Effects of swimming exercise on red blood cell rheology in trained and untrained rats, $J$ Appl Physiol 88 (2000), 2074-2080.

[98] O. Yalcin, A. Erman, S. Muratli, M. Bor-Kucukatay and O.K. Baskurt, Time course of hemorheological alterations after heavy anaerobic exercise in untrained human subjects, J Appl Physiol 94 (2003), 997-1002.

[99] J. Zhao, Y. Tian, J. Cao, L. Jin and L. Ji, Mechanism of endurance training-induced erythrocyte deformability in rats involves erythropoiesis, Clin Hemorheol Microcirc (2012). DOI: 10.3233/CH-2012-1549. 